\title{
Rabbit gastric ulcer models: comparison and evaluation of acetic acid-induced ulcer and mucosectomy-induced ulcer
}

\author{
Jin Hee Maeng', Eunhye Lee', Don Haeng Lee ${ }^{1,2 *}$, Su-Geun Yang ${ }^{3 *}$ \\ ${ }^{1}$ Utah-Inha DDS and Advanced Therapeutics Research Center, Incheon, Korea \\ ${ }^{2}$ Division of Gastroenterology \& Hepatology, Inha University Hospital, Incheon, Korea \\ ${ }^{3}$ Clinical Research Center and NCEED, School of Medicine, Inha University, Incheon, Korea
}

\begin{abstract}
In this study, we examined rabbit gastric ulcer models that can serve as more clinically relevant models. Two types of ulcer model were studied: acetic acid-induced ulcers (AAU) and mucosal resection-induced ulcers (MRU). For AAU, rabbit gastric mucosa was exposed by median laparotomy and treated with bottled acetic acid. MRU was examined as a model for endoscopic mucosal resection (EMR). Normal saline was injected into the submucosal layer and the swollen mucosa was resected with scissors. Endoscopic mucosal resection (EMR) is frequently performed for treatment of early gastric cancers. This procedure inevitably leads to ulcers and bleeding. Bleeding control is the major concern in endoscopic mucosectomy, and some endoscopic hemostatic agents are currently under clinical and preclinical studies. MRU was developed as a model for these induced ulcers and the evaluation of the healing process. The clinical relevancy of those models was compared with that of rat models. Progressive healing was observed for 7 days based on histology. Rabbit models demonstrate round, deep ulcers with clear margins and well-defined healing stages that were difficult to define in rat models.
\end{abstract}

Key words: Gastrointestinal ulcers, bleeding, acetic acid, mucosectomy, rabbit

Received 2 April 2013; Revised version received 27 May 2013; Accepted 28 May 2013

Gastrointestinal (GI) ulcers are defined as a degenerative and necrotic breakdown of gastro-intestinal mucosa [1]. Physicians ascribe the causes of GI ulcers to Helicobacter pylori, alcohol intake, spicy food, drugs such as nonsteroidal anti-inflammatory drugs (NSAIDs), neoplasia, inflammatory bowel disease, chronic gastritis, aging, etc. [2-3]. Molecular intermediates such as inflammatory cytokines (TNF- $\alpha$, interleukins), cyclooxygenase, Noxidase and reactive oxygen species are involved in the progressive developments of ulcers [4]. Digestive enzymes (i.e., pepsin, pancreatic juice, hydrochloric acid and bile acids) also play an important role in the aggravation of ulcers [1]. Antibiotic eradication of Helicobacter pylori dramatically reduced the incidence of GI ulcers in young people, but the incidence rate in people over 55 years of age is steadily increasing. Ulcer-related bleeding is another clinical issue in ulcer treatment. Groenen et al. reported that $22.7 \%$ of patients (158 cases) showed signs of bleeding in 696 cases of duodenal ulcers; $12.3 \%$ of patients showed signs of bleeding in 487 cases of gastric ulcers [5]. The bleeding rate increases dramatically when older patients develop diseases like hypertension, diabetes and arthritis [6].

The acetic acid-induced ulcer (AAU) model, established 40 years ago, has been widely used as a laboratory animal model of chronic gastric ulcer [7]. Although other chemicals such as ethanol, indomethacin, ceramide, and NSAID have been selected for ulcer modeling, AAU is

\footnotetext{
*Corresponding authors: Su-Geun Yang, Department of new drug development and NCEED, School of Medicine, Inha University, 3ga, Shinheung-dong, Jung-gu, Incheon 400-712, Korea

Tel: +82-32-890-1183; Fax: +82-2-890-1199; E-mail: sugeun.yang@inha.ac.kr

Don Haeng Lee, Division of Gastroenterology \& Hepatology, Department of Internal Medicine, Inha University Hospital, 3-ga, Shinheung-dong, Jung-gu, Incheon 400-712, Korea

Tel: +82-32-890-2548, 2245; Fax: +82-32-890-2549; E-mail: Idh@inha.ac.kr
}

This is an Open Access article distributed under the terms of the Creative Commons Attribution Non-Commercial License (http://creativecommons.org/licenses/ by-nc/3.0) which permits unrestricted non-commercial use, distribution, and reproduction in any medium, provided the original work is properly cited. 
more clinically relevant for screening ulcer drugs and studying healing mechanisms [8]. Several methods of inducing AAU have been reported, such as submucosal injection method, intra-gastric injection method and gastric serosal application method. In the submucosal injection method, a small amount of acetic acid is injected into the submucosal layer of the anterior wall of the glandular stomach after exposing the stomach. In the same way, acetic acid can be injected through the gastric wall into the gastric cavity, and the ulcer size can be controlled through round-forcep clamping. But these methods do not control the rate of ulceration, and gastric wall adhesion commonly occurs in the rat model. To prevent gastric adhesion, acetic acid was applied to the serosal side, but this model is physically different from a real ulcer because erosion occurs in the gastric wall rather than in the mucosal layer. For these reasons, a model involving a larger animal like a rabbit might be more relevant for gastric ulcer.

Endoscopic mucosal resection (EMR) is another major cause of gastric ulcers and bleeding [9-11]. EMR is widely performed for removal of early gastric malignancy. Although EMR is a relatively non-invasive endoscopic surgery that allows rapid recovery without long hospitalization, EMR-related bleeding is observed in around $20 \%$ of patients and requires close observation after resection [12-13]. Around 4-14\% of patients after EMR experience severe bleeding and receive a blood transfusion [4,14]. The degree of bleeding is highly dependent on the malignancy status and the physician's performance [15-16]. Ulcer-coating spray powder for the treatment of EMR-induced ulcers and bleeding is currently in clinical trials and shows beneficial efficacies [17-18]. More appropriate animal models for EMRrelated ulcers are required.

Most experiments for laboratory ulcer models use rodents. Mammalian stomachs share histological similarities between species, but they also possess irrefutable differences by species. The basal part of the glandular lamina propria of mice and rats is composed of a varying proportion of parietal and chief cells. Like humans, rabbits have a predominant population of chief cells on the base of the gastric wall [19]. Thus, rabbit shares more structural similarities with human.

The thickness of the rat gastric mucosa is one of the major pathologic factors for gastric ulcer modeling. Rat has 500 600 $\mu \mathrm{m}$ of gastric mucosa, which is unsuitable to obtain a uniform ulcer model in terms of size and depth [20]. Human mucosa has a thickness of around 1 $\mathrm{mm}$. In this respect, larger animals like rabbits and micro-pigs may be more relevant laboratory animals for gastric ulcer models. In this study, we used rabbit as a laboratory animal model for gastric ulcers. The gastric wall was surgically opened and treated with acetic acid. Similarly, mucosal resection was used to generate an EMR-related ulcer model. After surgery, rabbits were allowed to recover. Healing of mucosal defect was observed by histological observation for several days. Our study thus indicates that larger laboratory animals like rabbits are more proper and relevant to obtain a homogeneous model for gastric ulcers and a new ulcer model for EMR.

\section{Materials and Methods}

\section{Animals}

All animal care and experimental procedures were conducted in accordance with the guidance from the Experimental Animal Research Committee of Inha University (INHA 120622-145). A total of 41 female New Zealand rabbits, aged 10-11 weeks and weighing $2.1 \sim 2.5 \mathrm{~kg}$, were obtained from Orient Bio Inc. (Sungnam, Korea) and enrolled in this study. All rabbits were singly housed in cages (Daejong Instrument Industry Co., Ltd., Seoul, Korea). Autoclaved chow (Lab diet 5325, Orientbio Inc., Sungnam, Korea) and hyperchlorinated water were offered ad libitum. Eight-weekold male Sprague-Dawley rats $(\mathrm{N}=17)$ were purchased from Orientbio, Inc. (Sungnam, Korea). The rats were housed in cages with free access to feed (Lab diet 5053, Orientbio, Inc., Sungnam, Korea) and water. Room temperature and relative humidity were maintained between $22.2 \pm 1.1^{\circ} \mathrm{C}$ and $50 \% \pm 10 \%$, respectively.

\section{Rabbit acetic acid-induced gastric ulcer modeling (AAU model)}

The rabbit chronic gastric ulcer model using acetic acid was set up as follows. Rabbits were deprived of food for $20 \mathrm{hrs}$ before the operation but allowed access to tap water. Anesthesia was induced with an intramuscular injection of Rompun $(11.7 \mathrm{mg} / \mathrm{kg})$ in combination with Ketamine $(4.2 \mathrm{mg} / \mathrm{kg})$. Body temperature was monitored and maintained $\left(37\right.$ to $\left.38^{\circ} \mathrm{C}\right)$ with a heating pad throughout the experiment. The stomach was exposed by an approximately $7-\mathrm{cm}$ median incision on the middle line of the abdomen. The exposed stomach was 
cut open in line with the large curvature. The inner cavity of the stomach was opened slightly and washed with isotonic saline. Two ulcers were induced on opposite sides of the gastric cavity. Gastric mucosa in the upper corpus was osculated with bottled $60 \%$ acetic acid solution for $15 \mathrm{~s}$ and immediately rinsed with isotonic saline. The stomach and then abdomen were closed with sutures. After the closures, rabbits were injected with Cefazol inj. (Eagle Vet Tech Co., Ltd., Seoul, Korea) and UNI Ketopro (UNI Biotech Co., Ltd., Seoul, Korea) and replaced in cages. Animal chow was restrained for another $12 \mathrm{hrs}$. One, three and seven days after the operation, rabbits were sacrificed, and stomachs were harvested. The recovered stomachs were opened along the greater curvature, washed with isotonic saline and anteriorly spread open on a board.

\section{Rabbit endoscopic mucosal resection (EMR)-related ulcer model (MRU model)}

A rabbit EMR-related ulcer model was experimentally developed in this study. Gastric mucous layer was exposed by median laparotomy as described in previous experiments. $0.2 \mathrm{~mL}$ of isotonic saline was injected into the submucosal layer of the upper corpus. A diameter of $7-10 \mathrm{~mm}$ of the swollen mucosal layer was resected with a pair of scissors. Then the resected area was pressed with gauze to stop the bleeding and cleaned with sterile isotonic saline. After an antibiotic injection, the stomach and abdomen were sutured, and rabbits were introduced into individual cages. The stomachs were processed as described above.

\section{Rat acetic acid-induced gastric ulcer modeling}

Rats were fasted for $16 \mathrm{hrs}$ with free access to tap water before the operation. Anesthesia was induced in each rat with an intra-muscular injection of Rompun $(9.0 \mathrm{mg} / \mathrm{kg})$ in combination with Ketamine $(3.0 \mathrm{mg} / \mathrm{kg})$. Body temperature was monitored and maintained (37 to $38^{\circ} \mathrm{C}$ ) with a heating pad throughout the experiment. Two methods for generating chronic gastric ulcer were modified to the method described by Okabe et al. [8]. For intra-luminal application, $0.2 \mathrm{~mL}$ of $60 \%$ acetic acid solution was injected into the fundus, which was clamped with a ring forceps; the acetic acid was then removed after $45 \mathrm{~s}$. Two ulcers were induced on opposite sides of the gastric cavity. For serosal application, a vial containing $75 \%$ acetic acid was directly placed on the serosal surface of the rat stomach for $20 \mathrm{~s}$. The stomachs were rinsed with isotonic saline, the abdomens were sutured, and the rats were replaced in their cages. One or five days after the intra-luminal operation or serosal application, respectively, the rats were sacrificed and their stomachs were harvested. The recovered stomachs were opened along the greater curvature, washed with isotonic saline and spread open on a board.

\section{Determination of the induced-ulcer area}

Whole gross images were taken with scales, and ulcer area was calculated from the images by using an Image$\mathrm{J}$ program based on the images. Relative ulcer area was calculated as the ratio of the initial ulcer area. In the acetic acid-induced gastric ulcer rabbit model, the initial ulcer size was set as $2.88 \mathrm{~cm}^{2}$ based on the radius of the bottle orifice $(\varnothing ; 1.5 \mathrm{~cm})$. The inner diameter of the ring forceps was taken as the initial size of the ulcer in the intra-luminal application rat model.

\section{Histologic evaluation}

The recovered stomachs were assessed by histologic investigation. Specimens were rinsed with PBS buffer, dissected along the ulcer lesion and fixed in $10 \%$ neutral formalin. The fixed tissues were sliced longitudinally at intervals of 5-mm width, dehydrated in a series of graded ethanol and then embedded in paraffin wax. Paraffin blocks were introduced on a microtome and sliced in 4$\mu \mathrm{m}$ thicknesses; sections were mounted on slide glasses for hematoxylin and eosin (H\&E) staining. Stained sections were observed in bright field under a fluorescence microscope (DM2500, Leica, Germany).

\section{Statistical analysis}

All data are reported as meanSE. Statistical analysis was performed with Student's t- test. $P$ values of $<0.05$, $<0.01$, or $<0.001$ were considered statistically significant.

\section{Results}

Figure 1 and 2 shows that rabbit ulcer models were established very well by both methods. Representative images of the acetic acid-induced ulcer model (AAU) and the mucosal resection-induced model (MRU) showed very clear ulcer margins with deep defects of the mucous layer. Progressive healing of ulcers with time was defined very well in both models. In the acetic acidinduced ulcer models, the initial ulcer area was $288 \mathrm{~mm}^{2}$. The relative ulcer areas on 1, 3 and 7 days 


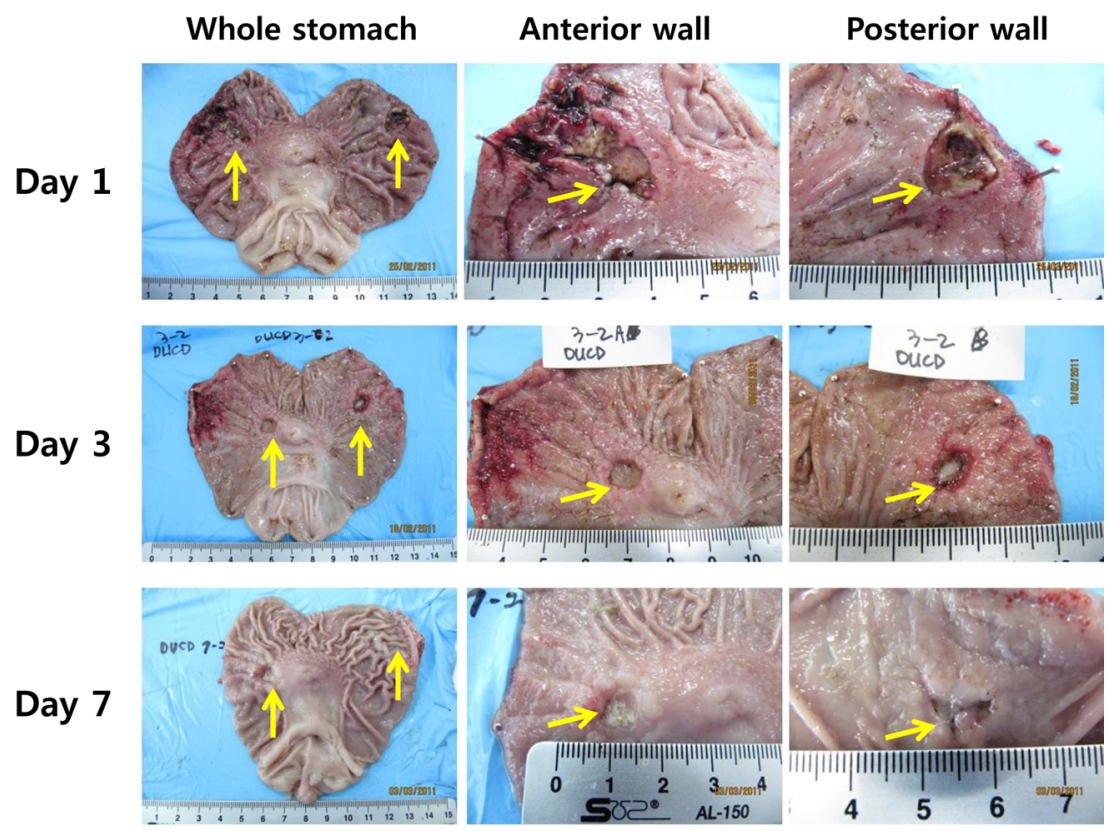

Figure 1. Acetic acid-induced rabbit gastric ulcer. An open glass bottle containing acetic acid was applied to the gastric mucosa for 15 seconds. Recovery of gastric ulcers was observed for a week.

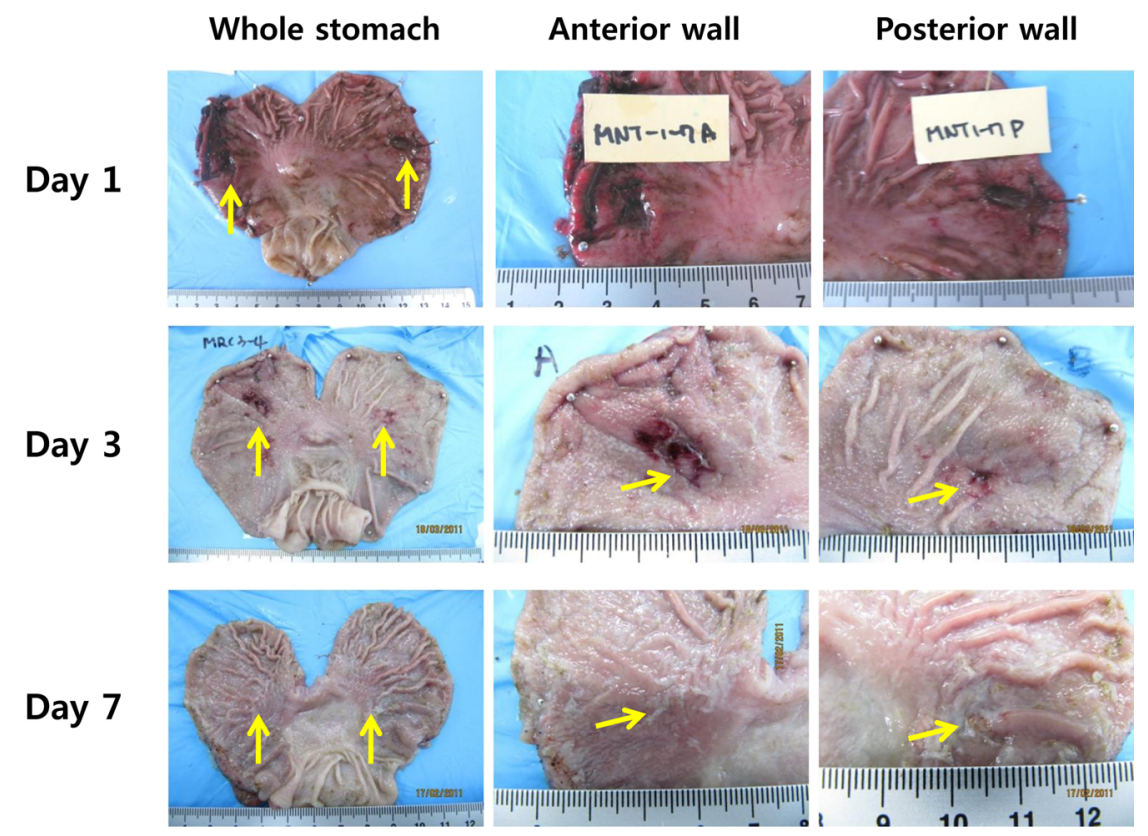

Figure 2. Mucosal resection-induced rabbit gastric ulcer. Isotonic saline was injected into the sub-mucosal layer, and the swollen tissue was resected following the typical endoscopic mucosal resection procedure.

after ulcer treatments decreased to $58.5 \pm 3.4 \%(P<0.001$ vs. initial ulcer size), $22.0 \pm 2.7(P<0.001)$ and $5.1 \pm 2.6$ $\%(P<0.001)$, respectively. In mucosal resection models, the initial wound area was $133.3 \pm 10.1 \mathrm{~mm}^{2}$ and the relative ulcer areas on 1,3 and 7 days after ulcer induction were $44.6 \pm 3.8 \%(P<0.001), 9.6 \pm 2.2 \%(P<0.05)$ and $2.2 \pm 1.7 \%(P<0.05)$, respectively. Interestingly, EMR- induced ulcers showed more precipitated healing than acetic acid models in terms of ulcer size.

Figure 3 shows rat ulcer models made by serosal application of acetic acid and intra-luminal injection of acetic acid with ring-forceps clamping, respectively. In our experience, intra-luminal injection of acetic acid generally caused total collapse of the gastric wall and 
A

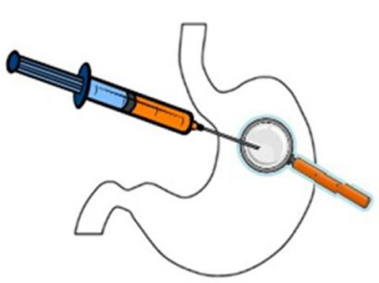

B

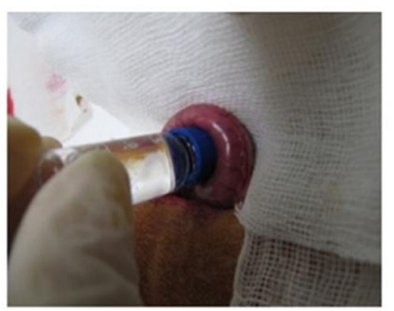

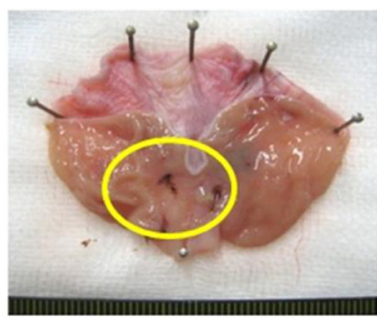

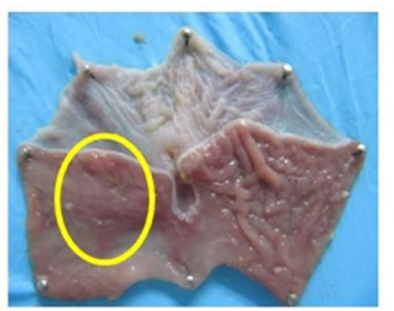

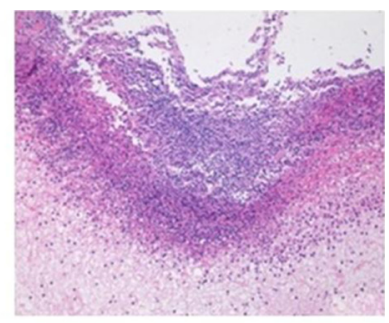

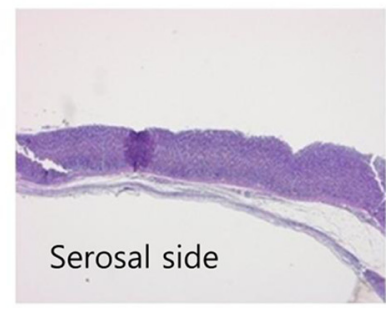

Figure 3. Rat experimental model of acetic acid-induced gastric ulcer. Ulcer margins and area are generally undetectable without microscopic observation. (A) Rat gastric ulcer induced by intra-luminal injection of acetic acid and its macroscopic and microscopic observation. Ulcer area is controlled by ring forceps. (B) Rat gastric ulcer induced by serosal application of acetic acid and its macroscopic and microscopic observation.

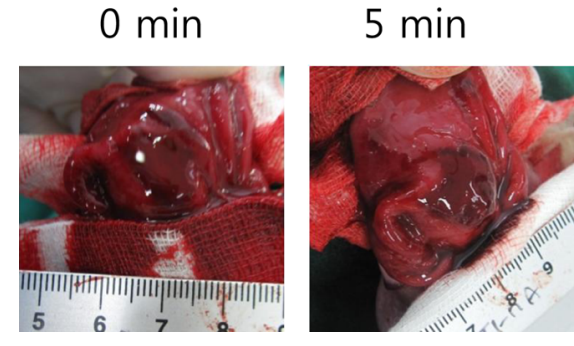

Figure 4. Mucosal resection-related bleeding in rabbit for development of endoscopic hemostatic agents.

adhesion of gastric tissues, and serosal application of acetic acid caused degradation of the muscular layer of the gastric fundus rather than of the mucous layer.
As mentioned before, the development of gastric hemostatic agent inevitably requires an gastric bleeding models. Our rabbit mucosal resection model accompanied mucosal-resection related bleeding and could be applied as appropriate model for hemostasis control (Figure 4).

In Figure 5, both models initially showed extensively damaged mucosa with diffuse and infiltrated hemorrhage. In particular, MRU showed total loss of the mucosa and partial loss of the submucosa layer. On day 3, partial recovery of mucosal structures was observed in both models. MRU showed more precipitated recovery of mucosal structures including muscularis mucosa, submucosa and cardiac glands on day 3 and increased mucosal thickness on day 7. Infiltration of inflammatory

\section{Day 1}

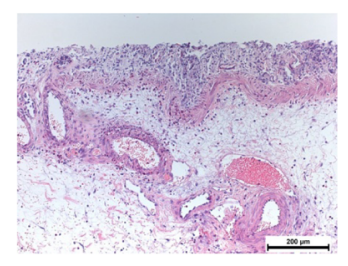

AAU

MRU

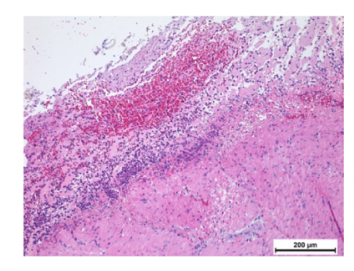

Day 3
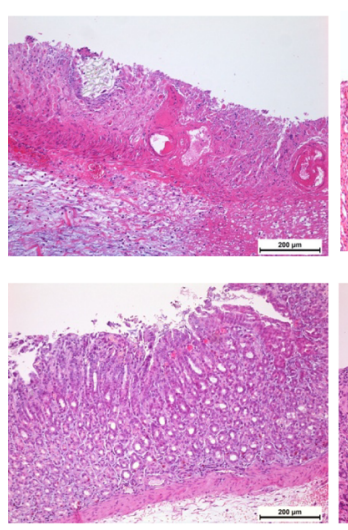

Day 7
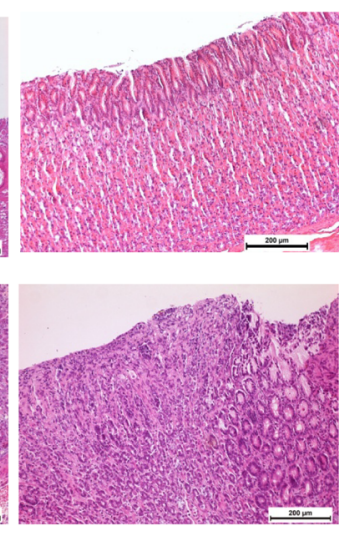

Figure 5. Microscopic observation of H\&E stained ulcer tissues. On day 1, total collapse of gastric mucosae was observed in both models (AAU and MRU). Then progressive recovery of mucosa layers (i.e., muscularis mucosa, gastric glands, mucosa columnar epithelium) was detected. Scale bar is $200 \mu \mathrm{m}$. 
cells was observed in AAU with delayed recovery of the mucosa. Mucosal thicknesses of AAU and MRU were $284 \pm 16$ and $685 \pm 45 \mu \mathrm{m}$, respectively, on day 3 . The normal mucosa has a thickness of $900 \pm 14 \mu \mathrm{m}$ based on our observations.

\section{Discussion}

Gastrointestinal ulcers are caused by diverse factors. In this study, we investigated the larger animal models rather than rodent models. Rabbit ulcer models were established via acetic acid treatment and mucosal resection method. Representative images of the acetic acid-induced ulcer model and the mucosal resectioninduced model showed very clear ulcer margins with deep defects of the mucous layer. Progressive healing of ulcers with time was defined very well in both models. Generally, rat ulcer models do not give a clear ulcer margin, as shown in Figure 3, and thus eventually require more extensive histological observations. Rat ulcer models were experimentally induced by several previously reported methods in our study. Figure 3 shows rat ulcer models made by serosal application of acetic acid and intra-luminal injection of acetic acid with ring-forceps clamping, respectively. Several other methods for rat gastric ulcer modeling have been developed. However, our data shows that directional contact of acetic acid with the gastric mucosal layer is the more relevant model for gastric ulcers, and this ultimately required stomach open surgery. To this point, rabbit, a larger animal than conventional rodents, is therefore a more relevant and reliable laboratory animal for the gastric ulcer model.

A decrease in ulcer size is the most conclusive data for screening antiulcer drugs. A clear ulcer margin allows easier determination of the ulcer size. Our data shows that the rabbit model is a simple model for the determination of ulcer size.

Endoscopic mucosal resection always leads to troublesome bleeding from the resected lesions $[4,14]$. Bleeding also accompanied our rabbit mucosal resection model, which could also serve as a bleeding control model for the development of hemostatic agents (Figure 4). Recently, endoscopic hemostatic agents, regarded as an emerging technology in this area, have improved clinical outcomes for several GI bleeding conditions [2122].

Our gastric ulcer models (AAU and MRU) initially showed extensively damaged mucosa with diffuse and infiltrated hemorrhage (Figure 5). In particular, MRU showed total loss of the mucosa and partial loss of the submucosa layer. On day 3, partial recovery of mucosal structures was observed in both models. Especially, MRU showed more precipitated recovery of mucosa including muscularis mucosa, submucosa and cardiac glands on day 3 and increased mucosal thickness on day 7. Infiltration of inflammatory cells was observed in AAU with delayed recovery of the mucosa. Those results corresponded with gross images (Figure 1 and Figure 2). In this study, histology revealed progressive healing of the gastric ulcer by the mucosal structures.

In this study, we selected rabbit as a laboratory animal model for gastric ulcers. Acetic acid-induced ulcers and mucosal resection-induced ulcers were developed in this study. Based on our observations, rabbits provide more relevant, reliable and simple laboratory ulcer models than small rodent models for estimating the ulcer healing process and screening of antiulcer drugs. Furthermore, rabbit mucosal resection typically reflects EMR-related bleeding and can be used for evaluation of endoscopic hemostatic agents. This model also showed that the healing process was differentiated by the induction model, which may be explored in future studies.

\section{Acknowledgments}

This study was supported by a grant of the Korean Health Technology R\&D Project, Ministry of Health \& Welfare, Republic of Korea (A111060).

\section{References}

1. Lam SK. Etiology and pathogenesis of peptic ulcer. J Gastroenterol 1994; 29(Suppl 7): 39-54.

2. Blaser MJ. Hypotheses on the pathogenesis and natural history of Helicobacter pylori-induced inflammation. Gastroenterology 1992; 102(2): 720-727.

3. Kurata JH, Haile BM. Epidemiology of peptic ulcer disease. Clin Gastroenterol 1984; 13(2): 289-307.

4. Ahmad NA, Kochman ML, Long WB, Furth EE, Ginsberg GG. Efficacy, safety, and clinical outcomes of endoscopic mucosal resection: a study of 101 cases. Gastrointest Endosc 2002; 55(3): 390-396.

5. Groenen MJ, Kuipers EJ, Hansen BE, Ouwendijk RJ. Incidence of duodenal ulcers and gastric ulcers in a Western population: back to where it started. Can J Gastroenterol 2009; 23(9): 604608

6. Adamu MA, Weck MN, Gao L and Brenner H. Incidence of chronic atrophic gastritis: systematic review and meta-analysis of follow-up studies. Eur J Epidemiol 2010; 25(7): 439-448.

7. Okabe S, Roth JL, Pfeiffer CJ. A method for experimental, penetrating gastric and duodenal ulcers in rats. Observations on 
normal healing. Am J Dig Dis 1971; 16(3): 277-284.

8. Okabe S, Amagase K. An overview of acetic acid ulcer models-the history and state of the art of peptic ulcer research. Biol Pharm Bull 2005; 28(8): 1321-1341.

9. Darbari A, Kalloo AN, Cuffari C. Diagnostic yield, safety, and efficacy of push enteroscopy in pediatrics. Gastrointest Endosc 2006; 64(2): 224-228.

10. Carmack SW, Genta RM, Schuler CM, Saboorian MH. The current spectrum of gastric polyps: a 1-year national study of over 120,000 patients. Am J Gastroenterol 2009; 104(6): 1524-1532.

11. Gotoda T. Endoscopic resection of early gastric cancer. Gastric Cancer 2007; 10(1): 1-11.

12. Ono H, Kondo H, Gotoda T, Shirao K, Yamaguchi H, Saito D, Hosokawa K, Shimoda T, Yoshida S. Endoscopic mucosal resection for treatment of early gastric cancer. Gut 2001; 48(2): 225-229.

13. Miyashita M, Tajiri T, Maruyama H, Makino H, Nomura T, Sasajima K, Yamashita K. Endoscopic mucosal resection scissors for the treatment of early gastric cancer. Endoscopy 2003; 35(7): 611-612.

14. Kojima T, Parra-Blanco A, Takahashi H, Fujita R. Outcome of endoscopic mucosal resection for early gastric cancer: review of the Japanese literature. Gastrointest Endosc 1998; 48(5): 550-554; 554-555.

15. Okano A, Hajiro K, Takakuwa H, Nishio A, Matsushita M. Predictors of bleeding after endoscopic mucosal resection of gastric tumors. Gastrointest Endosc 2003; 57(6): 687-690.
16. Yamaguchi Y, Katsumi N, Tauchi M, Toki M, Nakamura K, Aoki K, Morita Y, Miura M, Morozumi K, Ishida H, Takahashi S. A prospective randomized trial of either famotidine or omeprazole for the prevention of bleeding after endoscopic mucosal resection and the healing of endoscopic mucosal resection-induced ulceration. Aliment Pharmacol Ther 2005; 21(Suppl 2): 111-115.

17. Holster IL, Poley JW, Kuipers EJ, Tjwa ET. Controlling gastric variceal bleeding with endoscopically applied hemostatic powder (Hemospray ${ }^{\mathrm{TM}}$ ). J Hepatol 2012; 57(6): 1397-1398.

18. Giday SA, Kim Y, Krishnamurty DM, Ducharme R, Liang DB, Shin EJ, Dray X, Hutcheon D, Moskowitz K, Donatelli G, Rueben D, Canto MI, Okolo PI, Kalloo AN. Long-term randomized controlled trial of a novel nanopowder hemostatic agent (TC-325) for control of severe arterial upper gastrointestinal bleeding in a porcine model. Endoscopy 2011; 43(4): 296-299.

19. Ghoshal NG, Bal HS. Comparative morphology of the stomach of some laboratory mammals. Lab Anim 1989; 23(1): 21-29.

20. Svestka T, Krechler T, Zák A, Fabry TL, Zhang ZG. [Effect of fasting on gastric mucosa thickness: experimental study in laboratory rats]. Cas Lek Cesk 2003; 142(12): 751-754.

21. Holster IL, Kuipers EJ. Update on the endoscopic management of peptic ulcer bleeding. Curr Gastroenterol Rep 2011; 13(6): 525531.

22. de la Torre RA, Bachman SL, Wheeler AA, Bartow KN, Scott JS. Hemostasis and hemostatic agents in minimally invasive surgery. Surgery 2007; 142(4 Suppl): S39-45. 